\title{
Co-ordination and Specialisation
}

\author{
Massimo Egidi ${ }^{1}$ and Massimo Ricottilli ${ }^{2}$
}

\begin{abstract}
This paper addresses the problem of division of labour as an evolutionary process in which co-ordinated agents attempt problem-solving as a search in problem space. Co-ordination stress and learning trigger adaptation but as complexity rises radical solutions are sought prompting radical organisational as well as technological change. The analytical issues involved are further explored by resorting to the so-called NKC model of evolution and co-evolution.
\end{abstract}

\footnotetext{
${ }^{1}$ Department of Economics and Computable and Experimental Economics Laboratory, University of Trento, via Inama 1, 38100 Trento, Italy

${ }^{2}$ Department of Economics and Statistics, University of Trieste, Piazzale Europa 1, 34100 Trieste, Italy
} 


\section{Path-dependence in organisational learning}

Persistency of organisational diversity has received a considerable amount of attention in the recent literature, and the application of some mathematical tools such as Landscape Theory and Polya Urns in economic and organisational contexts has permitted new promising results in the attempt to explain it. An interesting progress into this direction has been accomplished when it has been suggested that sources of diversity can be found in learning processes and in connected selection pressures being characterised by path dependency and which may, therefore, give rise to a display of diverse organisational forms.

When we refer to learning we have to distinguish at least two different and opposite effects: on the one hand we have to consider micro-learning activity which is the most important force to stabilise behaviour into repetitive patterns and which gives rise to organisational inertia: internal rules of an organisation and the associated routinised behaviour are maintained and repaired via this activity. On the other hand, learning can be considered in its full and radical dimension: in this respect, it is the force which allows organisations to jump from one configuration to another, internally redefining their rules and behavioural patterns. It is this force which leads to radically new division of labour.

Lock-in phenomena are the cornerstone of this distinction. In some experiments, which we will briefly discuss below, different groups, exposed to a different context have been induced to discover different sets of behavioural rules: the discovery has shown high persistency, a sort of "imprinting" which induces two groups to react in very different ways when exposed to the same configuration. This lock-in effect explains organisations' "cognitive inertia". Our starting viewpoint is that a fruitful way to analyse the division of labour is to consider it a process of co-operative innovation. In consequence we recur to models extracted from biological and evolutionary contexts in order to represent this process.

\section{A general overview on routinised behaviours, problem solving and division of labour}

Routinised behaviour can be considered as the building block of division of labour, and our working hypothesis is that to explain how new forms arise we need to understand two important: organisational inertia and path-dependency. For these reasons it is useful to revisit carefully the notion of routinised behaviour. A consolidated tradition exists - in the domain of computation theory - which considers routines to be computable programmes which can be represented by a set of condition-action rules. It is obvious that in social sciences strong resistance can arise against considering humans involved in a routinised activity as pure automata. The question amounts to understanding to what extent it is possible to attribute routinised behaviour to human beings.

Psychological studies (Luchins, 1942) are quite important for the comprehension of routinised individual behaviour. As this literature has emphasised, this behaviour is 
based on "routinised thinking", i.e. on the automatic use of rules which allows individuals to save on mental efforts. To avoid any confusion, we make reference to routinised behaviour (instead of routines) in order to indicate complex collective repetitive patterns of actions. The point at issue here is to see to what extent the representation of human behaviour in terms of rule-based activities can be used in the context of co-operation and co-ordination among different individuals. Consider the following points.

1. We must accept that in a co-operative context a micro-learning activity is normally at work, and consider situations in which an individual's activity is fully routinised, i.e. covers all possible contingencies with memorised set of rules, as an extreme case. Only in these extreme situations behaviours are fully mechanised, and the learning process is inactive.

2. Call "co-ordination rules" the rules which embody mutual relationships, i.e. prescribe actions which are compatible with the actions performed by the partners. Co-ordination rules are largely interiorised by individuals, who do not need detailed and specific orders to realise the co-ordinate task. If an unexpected condition arise, it follows either a conflict among rules or the lack of rules, and co-ordination fails. A machine cannot work in these conditions, while men's reaction is typically to find a solution, i.e. start learning and exploring in the space of rules.

3. The previous observations suggest that a fully routinised activity is possible, or at least more likely to happen, when tasks are performed by isolated individuals (Weisberg (1980)). In co-ordinated processes, in fact, purely routinised behaviours would require taking into account other actors' actions, including errors and conflicts, which require deliberation and learning: interactions normally are exponentially increasing with the number of participants.

4. Purely routinised collective behaviours are rather difficult to realise because, to cover any possible contingency, they require a huge set of rules governing the interactions among actors: this is the equivalent of complete markets with rational expectations in general equilibrium theory. We claim that there are limits to the complexity of the set of rules that can be activated by boundedly rational actors. Beyond this threshold, reasoning cannot be substituted by purely automatic behaviour.

March and Simon (1958) claim that the process of division of labour can be modelled as a process of collective problem solving ; in fact problem solving activity can be represented as search in problem space; and the search process is normally realised by means of a recursive division of problems into sub-problems the outcome of which is division of labour. We add two observation to this viewpoint : First, this view implies that the outcome of a problem solving process performed by a team is not only a new division of labour but also a new way to co-ordinate the divided parts; coordination is the complementary part of the division of labour which follows the 
realisation of a project; the further the division of labour proceeds, the more the different divided parts require co-ordination and the more information becomes dispersed. Second, this process is not deterministic, but highly conjectural and uncertain. In fact when a problem has been decomposed in a set of sub-problems, generally not all of the sub-problems will be immediately solvable and, consequently, they will, in turn, be decomposed into simpler ones. Decomposition then recursively proceeds until all the "relevant" sub-problems have been solved. The procedure "by decomposition" is therefore a conjectural one : a) there is no set of decomposition rules which a priori allows agents to achieve a certain result and b) agents can verify the solvability of the original problem only when all the "relevant" sub- problems have been solved.

This way of solving problems is characteristic of projecting and planning activities inside organisations: first a task is drawn up, on the presumption that is feasible, then the main task is decomposed into different functional parts. This division proceeds recursively until every part of the project has been recognised as feasible.

\section{The observational and experimental level}

If we assume individuals as capable of forming an internal mental representation of the situation, based on symbols and their manipulation, experiments must be devised to confirm or refute this approach not only at the behavioural level but also at the level of involved mental models. This can be done, beyond the experiments on the Einstellung effect, with a sophisticated use of the methods of experimental psychology. It is useful to emphasise that this level -rule interiorisation, memorisation, etc. - is at least partially observable. Problems in classical Expected Utility theory began when, with the experiments of Maurice Allais, and later of Kahnemann and Tversky, it became possible directly to confirm or refute a large number of propositions claimed by the theory. The same holds for the empirical analysis of other mental activities involved in human behaviour, like reasoning and problem solving. Therefore the more the methods of experimental psychology advance, the easier is to verify on empirical grounds whether routinised behaviour in the real world is or is not rule-based, and more generally to what extent individuals act on the basis of an internal model of the world. One of the limits of the standard psychological method of experimentation is that it is based on direct individuals' responses (see for example protocol analysis), and therefore does not take into account knowledge partial opaqueness and actors' partial unawareness.

One alternative experimental device, which allows experimenters to move beyond these limits by taking into account the opaqueness of individual's knowledge, has been realised by creating an artificial context for collective action: a computer game in which many agents must cooperage to achieve a common goal, without being submitted to verbal tests. This artificial game has been created by Cohen and Bacdayan (1994) and permits to explore the emergence of rules of co-ordination without involving players' verbal competence. Since the authors provide a detailed description of the rules of the Target the Two (TTT) game, it will only be briefly introduced here. The deck consists 
of 6 cards: $2 * 3 \vee 4 \vee$ all six cards are used in each game. Each player has one card and the other four are placed in four positions on the board named Target, Up, DownC and DownN (see figure 1).

According to the rules of the game the problem is solved when $2 v$ is placed in the Target position by one of the players. This is the final goal and all the configurations with $2 \checkmark$ in Target are possible final configurations of the board. In order to solve the game and put $2 \checkmark$ in Target, players must take a series of intermediate steps. In the configuration shown in Figure 1, for instance, one of players must find $2 \boldsymbol{v}$; moreover, since neither of them can exchange $4 *$ with $2 \bullet$, they must place another card in Target which allows the final exchange with $2 \vee$. For each configuration it is possible to identify sequences of intermediate steps that must be accomplished to solve the game. These sequences of intermediate steps can be conceived of as a decomposition of the problem of the game ( $2 \bullet$ in Target) into sub-goals.

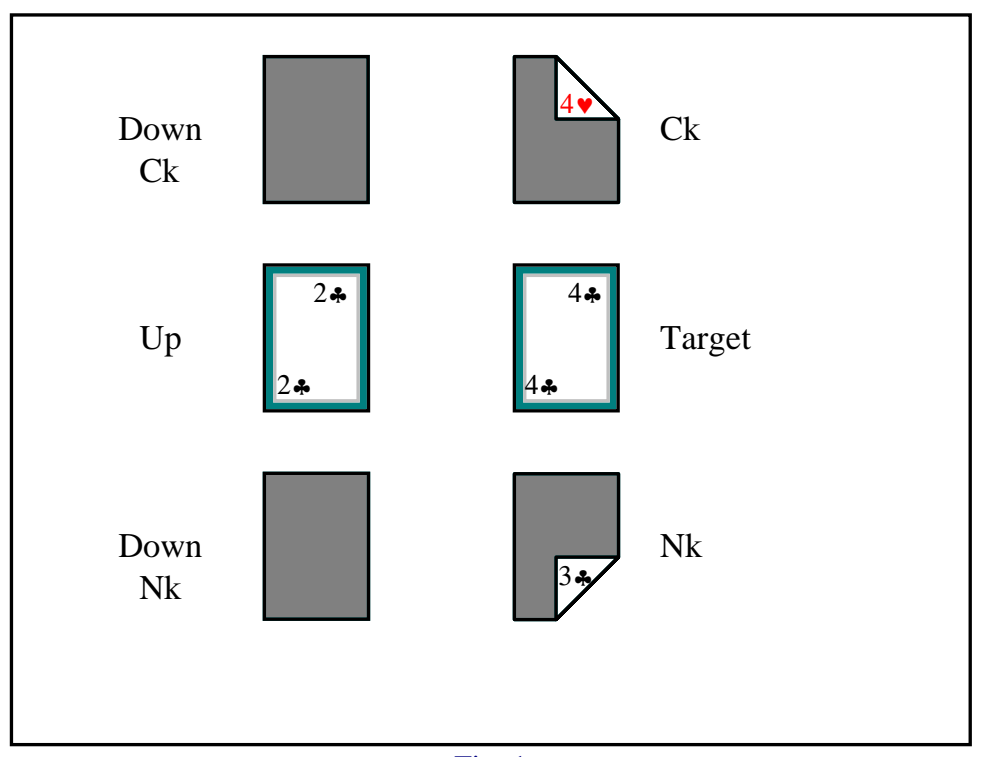

Fig. 1

A graph (see figure 2) has been introduced in order to represent the space of subgoals for any possible configuration. This representation focuses only on the card occupying the Target area and illustrates all possible transitions in the Target area [Egidi 1994 pages 9-11]. Note that a change between two configurations of the Target marked by horizontal lines is legally performed only by Numberkeepers, while a change of configurations marked by vertical lines is available only to Colorkeepers. By using the graph of sub-goals represented in Figure 2 it is possible to follow the progress of the solution to a game and to keep track of the sequence of cards occupying the Target area from the initial board to its solution. Reasoning backwards and given the rules of the game, the only possible configurations that immediately precede the final one (that is $2 \checkmark$ in Target) are those in which the Target position is occupied by either $3 \boldsymbol{\varphi}$, or $4 \boldsymbol{v}$ or 2*. In fact, because of the rules constraining the exchange with the Target, Colorkeeper can end the game by exchanging his card (that is $2 v$ ) with the card in Target only if 
they are of the same suit (that is $3 \vee$ or $4 \vee$ ). On the other hand, Numberkeeper is bound by the "number" constraint, and if he has $2 \checkmark$ in his hand and wants to end the game, the exchange will be possible only if the card in Target is 2*. Referring to Figure 2, the final goal is in the lower right corner. The sub-goal to be accomplished in order to achieve the final goal is to fill the Target position occupied by one of the cards that in Figure 2 are immediately close to $2 v$. Inspection of the graph of sub-goals clearly shows the decomposable nature of the problem; in fact, if the Target is occupied either $3 *$ or $4 *$, it is necessary to put in Target either $3 \boldsymbol{v}$, or $4 \boldsymbol{v}$ or $2 *$ and only afterwards try to end the game. We call $2 *$ the Colorkeeper's key-card, whereas $3 \varphi$ and $4 \boldsymbol{v}$ are named Numberkeeper's key-cards. In general, the key-cards are the cards that a player can exchange with a Target area occupied by either $3 *$ or $4 *$ in order to solve the game.

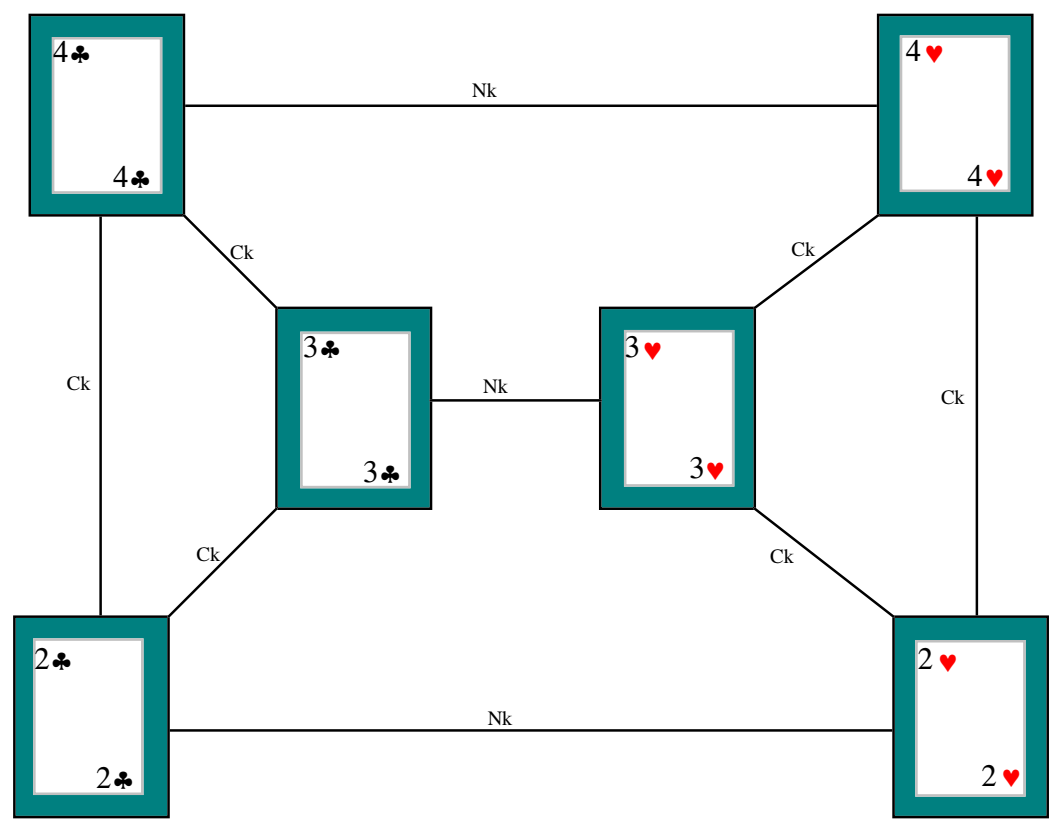

Fig. 2

From an analytical point of view, by keeping track of the sequence of cards placed in the Target position, it is possible to record the solution of the game in terms of the sequence of sub-goals and goal that the pairs of players follow to solve the game. For instance, the string $4 * 2 *$ identifies a specific path in Figure 2 and a well defined solution to the game: when the cards are dealt, the card in Target position is 4\%, Colorkeeper eventually exchanges his card (2*) with the Target, leaving Numberkeeper to end the game. On the other hand, these paths may be also very long (e.g. $4 \propto 3 \& 3 v$ $4 \bullet 4 \div 2 * 2 \vee)$.

It is interesting to note that a division of labour gradually emerges. To see this, notice that a strategy can be represented in summary form as a path in a sub problem graph; or as a set of rules to trigger an action for every game configuration. With regard to the TTT game, the 422 and 442 strategies can be described as paths in the problem space; any possible path in the graph defines a sequence of sub-goals to be achieved in order to solve the problem. This representation of a strategy does not mention specific 
moves, but gives the players directions about the goal to be achieved at each step There are many different ways to realize the goals corresponding to every path in the graph. For example, suppose that Colorkeeper plays a 422 strategy; he must search for his keycard (2*) and put it in Target area. In relation to the card distribution, 2* may be in many different (covered or clear) positions on the board, and therefore the sequences of actions corresponding to the same goal may differ considerably. As it is clear from the last of the two figures, there are two different strategies to realize the goal when the card in Target position is $3 *$ or 4 \&, strategies which we have called 442 and 422 respectively.

\begin{tabular}{|c|c|c|c|c|c|c|c|c|c|}
\hline & \multicolumn{5}{|c|}{ COLORKEEPER } & \multicolumn{4}{|c|}{ NUMBERKEEPER } \\
\hline & \multicolumn{3}{|c|}{ Conditions } & \multicolumn{2}{|c|}{ Actions } & \multicolumn{2}{|c|}{ Conditions } & \multicolumn{2}{|c|}{ Actions } \\
\hline & Hand & Up & Target & 422 & 442 & Hand & Up Target & 422 & 442 \\
\hline 1 & $2 *$ & $2 v$ & $4 \%$ & & & $4 \bullet$ & $2 v 4 *$ & $\mathrm{U}$ & $\mathrm{T}$ \\
\hline 2 & $2 *$ & $4 \vee$ & $4 \%$ & $\mathrm{~T}$ & $\mathrm{C}, \mathrm{N}$ & $4 \bullet$ & $2 * 4 *$ & $\mathrm{C}, \mathrm{N}$ & $\mathrm{T}$ \\
\hline 3 & $2 *$ & $\#$ & $4 *$ & & $\mathrm{C}, \mathrm{N}$ & $4 \bullet$ & \# 4* & $\mathrm{C}, \mathrm{N}$ & $\mathrm{T}$ \\
\hline 4 & $2 \bullet$ & $2 *$ & $4 *$ & & $\mathrm{P}$ & $2 \bullet$ & $4 \bullet \quad 4 \%$ & $\mathrm{P}$ & $\mathrm{U}$ \\
\hline 5 & $4 \bullet$ & $2 *$ & $4 *$ & & $\mathrm{C}, \mathrm{N}$ & $2 *$ & $4 \bullet \quad 4 \%$ & $\mathrm{C}, \mathrm{N}$ & $\mathrm{U}$ \\
\hline 6 & $\#$ & $2 *$ & $4 *$ & & $\mathrm{C}, \mathrm{N}$ & & $4 \bullet \quad 4 \&$ & $\mathrm{C}, \mathrm{N}$ & $\mathrm{U}$ \\
\hline 7 & $2 \bullet$ & $4 \bullet$ & $4 *$ & $\mathrm{C}, \mathrm{N}$ & $\mathrm{P}$ & $2 \bullet$ & $2 * 4 *$ & $\mathrm{P}$ & $\mathrm{C}, \mathrm{N}$ \\
\hline 8 & $2 \vee$ & $\#$ & $4 *$ & $\mathrm{C}, \mathrm{N}$ & $P$ & $2 \bullet$ & \# 4* & $P$ & $\mathrm{C}, \mathrm{N}$ \\
\hline 9 & $\#$ & $2 \vee$ & $4 \%$ & $\mathrm{C}, \mathrm{N}$ & $\mathrm{U}$ & $\#$ & $2 \bullet \quad 4 \%$ & $\mathrm{U}$ & $\mathrm{C}, \mathrm{N}$ \\
\hline 10 & $4 \bullet$ & $2 \varphi$ & $4 *$ & $\mathrm{C}, \mathrm{N}$ & $\mathrm{U}$ & $2 *$ & $2 \bullet \quad 4 \%$ & $\mathrm{U}$ & $\mathrm{C}, \mathrm{N}$ \\
\hline 11 & $4 \bullet$ & $\#$ & $4 \%$ & $\mathrm{C}, \mathrm{N}$ & $\mathrm{C}, \mathrm{N}$ & $2 *$ & $4 *$ & $\mathrm{C}, \mathrm{N}$ & $\mathrm{C}, \mathrm{N}$ \\
\hline 12 & $\#$ & $4 \bullet$ & 4 & $\mathrm{C}, \mathrm{N}$ & $\mathrm{C}, \mathrm{N}$ & $\#$ & $2 *$ & $\mathrm{C}, \mathrm{N}$ & $\mathrm{C}, \mathrm{N}$ \\
\hline 13 & $\#$ & $\#$ & $4 \%$ & $\mathrm{C}, \mathrm{N}$ & $\mathrm{C}, \mathrm{N}$ & $\#$ & $4 \%$ & $\mathrm{C}, \mathrm{N}$ & $\mathrm{C}, \mathrm{N}$ \\
\hline
\end{tabular}

The experiment shows that if players use the same strategy, let say 442, whatever the starting configuration, they perform in a suboptimal way in respect to "rational" players who resort to both strategies optimally in accordance to the specific initial configuration. Indeed, players become increasingly routinised and use the same strategy. The typical feature of the two routinised strategies is that they disentangle players' action while keeping cooperation active : in fact, players cooperate without using any information regarding their partner's actions as they appear from the board since strategies embody cooperation. Consider, finally, the table above which sets out the two players' condition action system.

This table fully defines the trait combination composed by the two players in the sense that among a large variety of different alternative strategies, the table restricts their choice to the two sub-optimal ones. The problem is to identify, within each table, subsets of condition action strings which can be modified independently from the rest. The number of independently definable subsets which compose the table is analogous to the epistatic number in Kauffmann' model. 


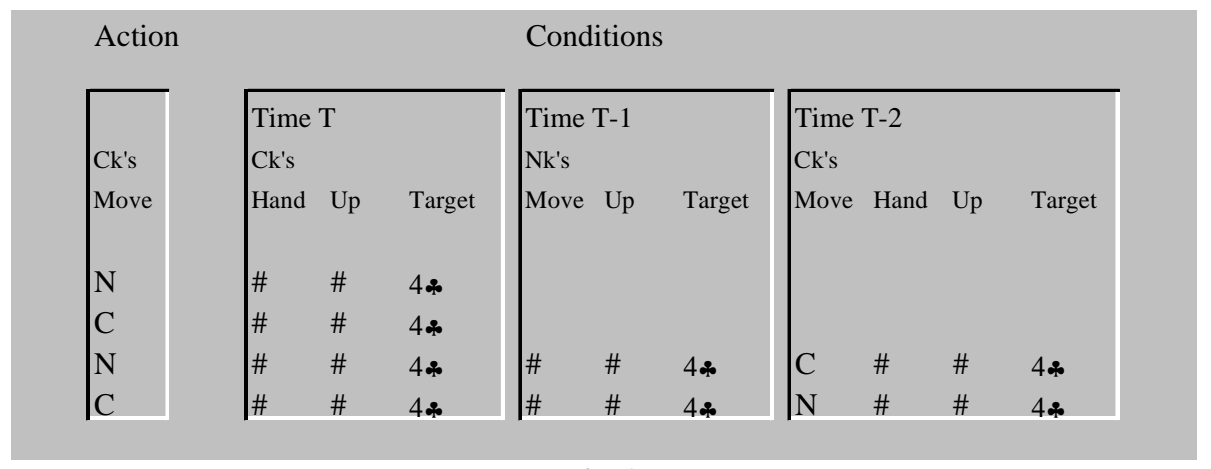

Fig. 3

Just to give a precise example, consider situation 13 in the table above : Ck must search a key card in order to accomplish his goal: the "subprogram" for search is a subset of rules as described in figure 3 .

It is clear that a local change in the subroutine described above, for example by changing in the first row $\mathrm{P}$ instead of $\mathrm{N}$, completely modifies the performance and the capacity of the routine to fulfil the goal : in fact in this case the search process is no longer complete and becomes therefore fully inefficient. Nevertheless, the other subroutines maintain their efficiency and do not have to be modified in order to improve the search process efficiency. This example gives, on the one hand, a clear idea of independence in the subset of rules belonging to an actor's set. On the other hand, it becomes clear that an epistatic link arises between different players ; in fact, if player A is unable to modify his search routine, player B can modify his own search process, by including a new part to help A. This part prescribes simply to search and pass (by putting it in the Up position) the card which is useful for the partner.

In summary, to achieve the final goal each player must discover his sub-goals, and must co-ordinate his action with the partner's one. The game therefore involves division of knowledge and co-operation among players, and gives rise to the emergence of organisational routines. The game admits multiple sub-optimal solutions: a number of different, locally optimal strategies, displayed in a rugged "landscape" of different rewards (Egidi and Narduzzo, 1996).

\section{Learning and innovation as an adaptive strategy}

Experimental evidence about co-ordination and division of labour in artificial contexts gives us interesting suggestions: largely non deliberate processes of distributed organisational learning can take place within organisations, but there are evident limitations to the possibility of learning co-operatively when the number of individuals involved increases. Experiments suggest that beyond a certain limit, co-operating requires a co-ordinating institution, for instance decisional hierarchies, or any coordinating device. Can the features we have now sketched be captured by some more formal model ? We suggest that the basic features of NKC Models (Kauffman 1989, 
Kauffman and Johnsen 1992) can provide a good basis to achieve this goal.

Even though this model has been developed mainly to study biological adaptation, its relevance can be considered quite general and applicable to economic analysis and organisational theory (Levinthal 1994). The basic assumption, which is critical but fundamentally befitting human behaviour, is that individuals are characterised by bounded rationality and that their problem solving activity can consequentially be viewed as a local search in problem space. The issue that a model such as the one under discussion is able to cope with is the increasing co-ordination difficulty encountered by organisations, namely firms, as they carry out their activity and are therefore called upon to face up to novel situations stemming from the very activity they perform. A most notable situation of the kind described is when organisations react by resorting to innovation. Activity in this context, for instance a functioning production process, can be portrayed as a co-ordinated ensemble of specialised tasks. Specialisation at each point in time is the outcome of problem solving which has led to specific knowledge. Slightly more formally, we can order these tasks as a sequence of $\mathrm{N}$ elements. As argued in more detail above, tasks cannot be completely routinised as an exhaustive and all encompassing programme fitting all states of the world. Learning takes place, partial routine repair occurs even in spite of strong hierarchical control; events which imply that tasks lend themselves to a manifold performance. Thus, each such task can be described by potentially different ways of execution, say under strong simplification, by $\mathrm{n}$ different ways.

Innovation is a deliberate attempt of an organisation to improve its performance. For simplicity's sake, we will assume a very simple pristine production process. Although, this view of a production system is entirely non-realistic and abstract, it may possibly be of help to focus on the argument to add a somewhat historical flavour to the case we wish to argue by imagining it as appropriate to a backward agricultural economy at a stage zero of development : a still very rudimentary, subsistence economy in which trade plays an insignificant role. In spite of its backwardness, this production system is an economically and technologically functioning set-up which requires specific knowledge and know-how (Ricottilli 1994). Seen in an evolutionary perspective, it can be viewed as a process which generates division of labour as well as implying it. Indeed, we may carry this observation further to assert that each production process appears at every point in time as the result of problem solving which has structured the task of producing something into specific phases. Each phase is, in fact, the outcome of decomposition or, to be more explicit, of a process which has broken down the original problem into sub-problems. It is the solution of such sub-problems to be organised within specific production phases. In this paragraph, we wish to point out some basic characteristics of the innovative search which takes place in such a framework. To do so, it is convenient to look upon the notion of extant technique as an organisational set up aimed at problem-solving by means of adequate problem-framing. As argued above, complexity and co-ordination difficulties warrant an organisational solution thanks to which sub-problems, or sub-tasks, may be more appropriately addressed in relative independence so as to focus at a given, more or less specific, partial production target rather than to the whole task. Thus, a technique of production, no matter how rudimentary and primitive, appears as a decomposed process, as a 
sequence of relatively independent phases. If we consider a process of production, the fact that it has been broken down into relatively well defined phases is the consequence of an in-house division of labour. Although, each phase need not necessarily appear to reflect a specialised body of knowledge, for phase decomposition may simply respond to the need of shortening a given task to minimise blunders, it is nevertheless true that through evolution independence and, more to the point, independent searching engender knowledge specificity. Thus, while at the outset of the decomposition process, phase specificity owes mainly to mere co-ordination and to the need of eschewing limiting constraints, as independence is strengthened and as it becomes more entrenched, each phase becomes more and more specialised with its own peculiar body of patent and tacit knowledge, its tools, procedures and above all routines. In this sense, division of labour generates division of knowledge.

Once a definition of technique currently in use is thus postulated, we may then simply describe each phase as a collection of traits each of which takes a particular form out of an empirically defined set. If we view even an extremely simple, pristine economy through these lenses, its dynamics appear nested into three different problematic layers. The first is, as a consequence of the phase independence postulate, an evolutionary problem confined within the boundaries of each phase. The second is, given phase specificity, an inter-phase co-evolutionary problem. The third amounts, by lifting the analytical plane still higher, to the co-evolution among phase-structured production processes or possibly firms within the economy. Let us take each of these different analytical planes in turn.

The evolutionary process within a simple phase In each phase there are, in general, $\mathrm{N}$ traits each of which comes with A characteristics fully describing the technique being operated. A combination of the $\mathrm{N}$ traits, a sequence, is thus such a description. An example may clarify this point. In phase $\alpha$ of a given process, the elements which are apt to define the technique being used are the following traits: (i) one machine, (ii) one type of labour and (iii) a co-ordination procedure: $\mathrm{N}=3$. Each one of these traits is currently present with a given characteristic: (i) the machine is of type $a$, (ii) labour is 'unskilled', (iii) the co-ordination procedure is 'top-down'. To any such description corresponds an action, in this case a co-ordinated, collective action leading to the execution of the tasks prescribed by phase decomposition. This bears a strong analogy to the condition-action execution of TTT-like games. Two important points must be made at this stage of the argument. Each trait is related to all other traits by a complementarity relationship. The reason is straightforward: in terms of the example which is made here, it is quite obvious that the kind of machine currently being used conditions the type of labour being employed and vice-versa. The co-ordination rule, in turn, is consistent with both. Thus, the performance of each trait, its contribution to the whole, whether identifiable as such or not being immaterial, strongly depends on all other traits. Given this, it is generally possible to establish overall performance in terms of a productivity indicator as a consequence of the technical and organisational characteristics in place. Each technique in each phase has, in this sense, a pay-off and is the result of an evolutionary process in which change has occurred. Given this environment, the general rules of the game with its legal possibilities or the body of knowledge, we can in abstract postulate that existing traits lend themselves to a finite 
number of changes. These changes are predictable in the sense that knowledge is discovered and acquired, as long as search and learning remain local, on a trajectory. Machine $a$ can change into machine $b, c, d$ etc. Unskilled labour can become skilled of type $\alpha, \beta, \gamma$ etc. Co-ordination can switch from a top-bottom to a bottom-up principle. Thus, the $\mathrm{N}$ traits can actually appear in terms of a finite number of characteristics, let $\mathrm{A}$ be such a number.. Each combination of traits bears a pay-off, that is to say a productivity performance in the stage in which it occurs. This pay off and its probability are unknown to searching agents, a direct consequence of their bounded rationality: they must, therefore, explore the various combinations in order to strike a positive outcome. This proposition is a statement on innovative activity. What triggers such an activity, at least at this stage of our analysis, will not be discussed in detail. Economic and behavioural literature abounds with theoretical and empirical recounts which uphold the view that it is a process which is strongly endogenous to economic activity. We now wish to discuss some formal characteristics. The searching problem is formally analogous to the TTT game: at any given time, agents are faced with a condition- action situation. Their performance is the outcome of the action taken when faced with the production conditions set by technique and other environmental conditions. Such an action is not necessarily predetermined in an unique way: variance occurs and learning takes place in consequence. It is clearly possible to draw a landscape by connecting points corresponding to combinations of traits.

Each trait combination is a technique and a corresponding action. They are both conducive to a performance which depends in essence on the way traits are combined and action taken. Whether the contribution of each trait with the taken characteristic can be identified or not remains an open question. For neo-classical theory factors of production contribute according to their marginal productivity. In this context, such a coupling between specific traits and their particular performance contribution is not necessary. If complementarity and interdependence are postulated, then pay-off change whenever traits take on a different characteristic. In the NKC model, fitness changes randomly: Each trait is randomly assigned a fitness value by a draw out of the unit distribution; this value changes not only when the trait in question takes on a new characteristic but also whenever traits to which it is linked through epistasis change. In both cases, new fitness values are re-attributed by a new random draw. In ours, performance, which is the equivalent of biological fitness, varies because innovation in other traits cause imbalance in the structure of complementarities: limiting constraints, mismatch of technical requirements, lack of co-ordination but also positive spill-overs, learning and scale effects are all factors which either lower or enhance performance in a deterministic sense. Thus, given a trait combination and assuming across-the-board interdependence between traits, an innovative change in any of them which would normally mean improvement per se, may change overall performance in any direction. Which direction it turns out to be is largely an empirical matter. Since each trait combination carries a pay-off and given that the latter changes completely even when a single trait is modified, it follows that the resulting landscape is likely to be very rugged.

The nature of searching on a landscape. In the context of a learning process, searching is the means by which innovation is realised. An organisation attempts to 
bring forth improvements. Likewise, in the TTT game, players attempt to find out a better sequence of moves, that increases the pay-off, until a strategy is consolidated. The former does so by altering its traits and by so doing it 'emits' information. Searching, however, does not escape the nature of activity as it results from past learning and the ensuing emergence of routines and cognitive inertia. It is, in fact, the latter that limits the range of searching to an adaptive process unless a major co-ordination effort lead it toward a long jump. Uncoordinated search is adaptive in the sense that cognitive inertia allows only for localised improvements, and for agents to focus on changing one element among the many making up the organisation at any point in time. Adopting the approach elaborated by theorists of rugged landscapes, searching is local and such as to explore neighbouring alternatives, organisational set-ups in the case of production processes or firms and close sequences of moves in the case of the TTT game. This statement calls forth a more precise notion of neighbouring alternatives. Again, the notion of neighbour in the NKC model proves useful.

1. A neighbour in this context is a 'genotype' or more generally a string of traits which differs for a characteristic of only one trait (a trait exhibiting a differing allele). Now consider a typical economic problem. Take the technical-organisational set-up of a production phase. A neighbour is one such set-up differing from any given one for the characteristic of only one of its elements. (for instance: machine type $a$, 'unskilled labour', 'top-down' organisational principle). Local search is an attempt to discover designs that, because of specific knowledge and cognitive inertia, are quite close. Each agent (or narrowly defined groups of persons) searches independently 'around' the object that he or she controls, operates, manipulates or otherwise handles: thus when he comes up with a discovery, quite likely it is the only person to do so.

2. This proposition may be rendered in slightly more formal terms. At any point in time, it is the discovery of only one characteristic to have the highest probability of occurring. If: $p_{i}$ is the probability of an innovation in element $i$. then the probability of a change of all elements is $\prod_{i}$ pi $\left\langle p_{i}\right.$. This result holds if the probability of a trait change is the same for all traits $\left(p_{i}=p_{j}\right.$ all $i$ 's and $j$ 's). There remains the probability that $\prod_{j} p j$, for $\mathrm{j}<\mathrm{N}$, be greater than $\mathrm{p}_{\mathrm{k}}$ for $\mathrm{k}$ not in $1,2 \ldots \mathrm{j}$ ). It is, nevertheless, a different matter if search is not independent but co-ordinated. Co-ordinated search can make the probability of achieving an innovation on all or more than one element (trait) plausibly higher: if co-ordination is pervasive then the sum of probabilities applies.

Now consider the TTT game. Once rules are stated each player, when confronted with the first initial board tries to discover a sequence of actions which seems to be appropriate. How the discovery process takes place is a matter analysed in a huge literature, initiated by Simon (1957, 1963), (see also Simon and Newell (1972)) in which is suggested that one of the key mechanisms is the sub-goaling activity. Players try to identify partial goals and to select a better fitting one. It is clear that this activity has a large random component, due to the incomplete information available, and 
moreover to the limited ability of the players to process the available information. Therefore the selection of a better division of tasks is a process with some random features, and the same happens for the identification of the strategies to realise the tasks. Suppose that, given a starting board, each player identifies a provisional task and a correlated set of rules of actions. It is likely that the provisional identification of action rules, will be considered unsatisfactory. Therefore the division of tasks is changed - and in consequence the sets of condition-action rules are modified - in order to improve the performance of the players.

For any given board lay-out, new condition-action rules are explored and revised; players' activity is oriented to improve the fitness of their performance by modifying the set of action rules. It is this set which is relevant and which is explored on account of the fact that the game is repeated. Whenever similar boards appear, condition action leads to search for improvement (shorter sequences)

These considerations provide two behavioural rules:

Rule A. Search is carried out by sampling in turn possible combinations of traits and it is stopped as soon as an improvement is found; the initial combination is random or historically given. Less efficient combinations are turned down (no adoption).

Rule $B$. Search is carried out by exploring neighbouring combinations.

These rules together with the pay-off structure outlined above provide a formal method to assess exploration and innovative adaptation. Consider the following elements:

1. The number of combinations over which search may be conducted is

\section{$1.1 \mathrm{~S}=\mathrm{A}^{\mathrm{N}}$}

if each phase possesses a different number $A_{j}$, then

$$
1.2 \mathrm{~S}=\mathrm{A}_{1} * \mathrm{~A}_{2} * \mathrm{~A}_{3} * \ldots \ldots \ldots \ldots \ldots * \mathrm{~A}_{\mathrm{N}}
$$

2. A neighbouring combination is one which differs for only one trait. This proposition gives content to the fact that since rationality is bounded and given cognitive inertia, search is local. The number of neighbours which each combination of traits possesses is

\section{$2.1 \mathrm{~N}_{\mathrm{b}}=(\mathrm{A}-1) \mathrm{N}$}

Epistatic dependence must now be addressed. As argued above traits of a combination are far from being independent. If this were the case, however, innovation in one trait would not jeopardise the performance of the remaining ones. Thus exploration of neighbouring sequences of traits would identify more performing solutions. If a trait is changed (say a machine) and this is more performing, then the entire technique would feature a higher pay-off. By discarding worse solutions, the most performing technique and associated action would be found. This process is, indeed, very similar to genetic hill-climbing of the Kauffman model. Trait independence insures 
that if search takes place eventually it will lead to discover the optimal solution by one trait adaptive changes.

Proposition 1. Since each fitness value is independent and unless the chosen combination is already optimal (exhibiting the highest fitness value), beginning randomly from any of the potentially available combinations it is always possible to find a neighbour with a higher fitness by random search

Epistatic influence changes this proposition. Complementarity and interdependence are the rule within a phase: the performance contribution of each trait depends crucially on other traits'. Assume that those linked by interdependence are $H$, the following are the epistatically linked combinations.

\section{$3.1 \quad \mathrm{~S}_{H}=A^{H+1} \quad H+1 \leq N$}

The important point is now that each performance varies in accordance to how traits are combined. Furthermore there is no a-priori direction which this change is likely to take. The consequence for innovative evolution is far ranging. In the previous case, when independence was the rule, hill climbing from neighbour to neighbour was sufficient to attain the optimum trait combination. Now a technically or organisationally better trait may bring about worse overall results. Thus, by applying the rule of searching randomly among neighbours (one trait mutants in genetic models), the path towards improvements may sooner or later lead to a lock-in into a sub-optimal combination. The space of combinations may allow for an optimal one and for ones upping the score of the one just discovered but they all lie outside the neighbouring space of combinations. A standard result of rugged landscape analysis is that when epistatic interaction prevails, the potentially optimal combination may not necessarily be reached. Starting randomly, improvement may end up in a less than optimal situation, i.e. in a situation in which all neighbours have lower performance values than the one already achieved and innovative activity may get locked-in on a suboptimal performance peak depending on the search starting point. Path-dependency is a crucial and powerful factor: if the economy happens to be on a historically given technical and organisational set-up and on account of the necessarily local nature of the quest for alternatives, it may end up in very mediocre capabilities.

An interesting question is: how many local optima (lock-in peaks) are likely to exist. Take any point in the landscape. According to 2.1 such a point has $\mathrm{N}_{\mathrm{b}}=(\mathrm{A}-1) \mathrm{N}$ neighbours. The probability that such a point is a local optimum is the probability that it has the highest performance value among its neighbours; hence, there is one favourable case out of $(A-1) N+1$ possible ones:

\section{1 $\mathrm{P}_{\mathrm{o}}=1 /[(\mathrm{A}-1) \mathrm{N}+1]$}

Since there are $A^{N}$ such points, the number of expected local optima is the number of points times the probability that each is a local optimum:

$4.2 \mathrm{~N}_{\mathrm{o}}=\mathrm{A}^{\mathrm{N}} \mathrm{P}_{\mathrm{o}}=\mathrm{A}^{\mathrm{N}} /[(\mathrm{A}-1) \mathrm{N}+1]$ 
Note that $\mathrm{N}_{\mathrm{o}}$ is an increasing function of $\mathrm{N}$ since $A \leq N$.

Proposition 2. When epistatic dependence prevails, and especially in the case of $H+1=N$ (complete interdependence), the possibility of path-dependent, suboptimal lock-in arises. The probability of lock-in increases with the dimension of possible combinations: $\mathrm{N}$.

The case of process lengthening. Innovation has, so far, been discussed in terms of final productivity. As already argued in the introduction, this is not a general case. Innovation introduces firstly a re-elaboration of co-ordination, new organisational principles, and secondly new tools, machines, farm implements, more generally instruments and means of production which do not only replace obsolete ones but which increase the degree of mechanisation and must therefore be produced in appropriate and specialised production As usual, they are specific, are the result of a successful innovative search, are subject to learning, require particular production routines and thus define a specific and progressively more specialised phase of production.. The implication of deepening mechanisation or trait enrichment is straightforward: $N$ rises. Thus, if proposition 2 holds, the probability that this economy will get trapped in mediocre performance levels rises accordingly. The economy will not be able to cross over potentially more rewarding combinations as long as it remains trapped in suboptimal peaks. The strength with which this economy is capable to innovate and generate applied technical progress depends on many factors on which we shall not dwell upon here. As vigorously argued in the literature, technical progress is, at least in part, endogenous and therefore subject to self reinforcing mechanisms. Accordingly, $N$ rises in time. But as it does, so does the probability of lock-in on account of increasing epistasis. Note that with an increasing $N$, the number of search combinations rises,as 5) clearly shows. There is, of course, more to innovate and more to co-ordinate. At the same time, conflicting constraints also rise with $N, H$ increasing with it.

There are further consequences which need to be considered. The first arises if with increasing $N$, the outcome of innovative changes becomes increasingly random. If this is the case performance levels become a random variable. Thus, with $N$ sufficiently high, combination pay-offs can be taken to be distributed as a normal distribution with mean $m$ and variance $m / N$ : the central limit theorem applies. It can now be easily shown that, as fitness values become fully random on account of epistatic interaction, the value of local optima tend to regress towards the mean. The landscape becomes more and more uniform as $\mathrm{N}$ increases, combined search directions leading to more and more similar and progressively more mediocre results (Kauffman 1989). The second consequence bears upon co-ordination capabilities. Each phase in this production process is the result of problem decomposition into sub-problems: it is the outcome of an effort to reduce complexity. As $N$ rises, however, complexity increases and the task of keeping a co-ordinated phase more difficult. Thus, productivity increases which might otherwise be attained through the innovative effort are lessened owing to coordination failures. These consequences produce a powerful result: a general tendency towards mediocrity and similarity.

Co-evolution among phases. Since the production process is phase decomposed, co-evolution among phases must now be considered. The process discussed above, 
however, lays the ground for co-evolution among different phases. Again, the results set out below are mainly drawn from models of rugged landscapes reformulated to accommodate another kind of interdependence, or epistasis. As in the case of species which evolve on their own landscapes, here we are faced with the problem of phases in which condition action, rule based activity takes place and innovation is successfully sought. Each one of these phases is, as postulated above, operationally independent as a way to cope with the complexity of the whole. It is nevertheless influenced by other phases. There is, again, a straightforward case of inter-phase complementarity: the simplest example is the output of one becoming the input of another. Reciprocal influence can, however, be quite general whatever the nature of the linkage. The latter can, without loss of generality, be made more precise by observing the trait combination of each phase. Let for simplicity's sake confine their number to two. In each point in time, there is an extant technique and action taken in each one of them and, what is of equal importance, change is about to occur owing to learning and searching. Let phase $\alpha$ innovate by adopting a different characteristic in one of its traits (for instance machine $a$ is replaced by machine $b$ ); while this event bears consequences in terms of pay off within the phase in relation to its own internal epistasis, it is in fact a move on its own landscape, it also carries an impact upon the functioning of one or more of phase $\beta$ traits if and when they adopt a specific characteristic among the possible ones. By following the simple case proposed here, machine $b$ in phase $\alpha$ helps to produce an output that only highly skilled manpower can best handle in phase $\beta$. The latter, therefore, will exhibit a different overall productivity pay-off according to its labour force's skills and to its paired phase adopting machine $b$. Phase $\beta$ landscape is thus reshaped by movements of phase $\alpha$. This occurrence is in general reciprocal. As it can be seen, the type of influence and relationship being discussed in this context resembles closely that which players in TTT games have on each other: a move by one player sets up a specific board which restricts the other player's action. The implication is that learning is thus thwarted to take a path it might not otherwise have taken and a direction that locks-in in one rather than the other of the two canonical strategies. Returning to a production framework, these considerations prompt the statement that there normally is inter-phase epistasis which links up traits in combinations that the process of change, i.e. innovation, brings about. The intensity of this reciprocal influence is determined by the number of traits that are actually involved in epistasis. Let such a number be $C$. It is important to note that, once a dimension of epistatic influence has been established, the innovative movement by one phase impinges on the other's and vice versa. Thus, if by searching phase $\alpha$ has reached a local optimum on its landscape and is therefore lockedin, searching by phase $\beta$ may budge it out of the 'trap' shifting its performance on either a lower or higher level. The same applies to phase $\beta$. Hence, the relationship between the two phases hinges on the probability of each of reaching a lock-in peak and thus on the relationship between internal epistasis, as measured by $H$, and the external one as measured by $C$. Simulations run by Kauffman and Johnson 1995 provide some results which are very relevant to co-evolution through innovation They can be summarised in the following points. 
1. Given a time span and $H$ for $n$ phases matched pairwise ( $\alpha$ with $\beta, \gamma$ with $\lambda$ etc.), as external epistasis increases as measured by an increasing $C$, the proportion of pairs which lock-in into equilibrium decreases. This is an important point but, as mentioned above, movement can go both ways: performance can either improve or worsen. Agents within each phase continue searching and will accept only improved solutions ('fitter' combinations) only to witness their efforts being voided by the search conducted by agents in the other phase, or being enhanced, as the case may be. If phases were entirely operationally independent and therefore uncoordinated, movement would be random. A direction of change may be imparted only by strengthening co-ordination, i.e. by increasingly integrating phases. On a formal plane, integrating phases into one implies increasing $N$, the number of traits, and, as argued above, at the risk of worsening innovative efficiency and of bringing about a mediocrity stalemate.

2. As a consequence of the previous point, as $C$ increases average performance levels reached by co-evolving phases decreases. This result holds since as long as both continue in their attempts to change the more they will get, in a manner of speaking, in each other's way. Thus, if reciprocal influence is reasonably high $(C>1)$, performance levels when a lock-in equilibrium is reached are higher than in the period while searching goes on. Innovation, if looked at from this viewpoint, is a two-edged phenomenon: it is the condition for improvement but it may also involve the risk of losing and, in any case, of higher turbulence and adjustment.

3. If $C$ is high and so is the risk of being upset by 'partners', high $H$ phases, i.e. ones with strongly interrelated traits, feature higher mean performance during the period in which searching occurs before lock-in than low $H$ phases. This is because high $H$ means higher probability of locking-in and thus of being less exposed to the changes brought about by paired phases. Vice-versa, if $C$ is low, low $H$ phases fare better. Independence pays in terms of diminished exposure.

4. Low $H$ phases, when both reach equilibrium, exhibit higher performance levels than high $H$ ones, this being a straightforward consequence of the evolutionary mediocrity implied by high $H$. Yet, when $C$ is high, it is the high $H$ ones which fare better. This is the somewhat paradoxical result of the fact that mediocrity shelters from exposure.

5. The higher is the number of co-evolving phases the longer it takes to lock-in into a suboptimal peak: a mixed blessing given the uncertainty about the direction of change of performance levels. Furthermore, if innovation occurs randomly, a higher number of phases implies a drop in average performance levels; a clear indication that only co-ordinated efforts become acceptable.

These results apply equally well if analysis is extended to deal with co-evolving firms and production units. Co-evolution appears, in this analytical framework, as an uneasy relationship. 


\section{Division of labour and learning: final remarks}

The conclusion drawn above indicates that phases', or for that matter, firms' performance relies heavily on the relationship between trait interdependence within and without units which articulate division of labour. The measure of $H$ and $C$ and their relative magnitude are crucial in determining the pattern of co-evolution. If $H$ is too high in respect to $C$, if internal epistasis dominates, lock-in into mediocre performance is accordingly highly probable while the effects of the external linkage is too weak. If $C$ is too high in respect to $H$, the constraints which arise out of uncoordinated innovative efforts become increasingly costly and harmful. Striking a fine balance between the two factors is a matter for a fine tuning co-ordination between co-evolving partners. Dysfunction does, indeed, occur because of disarray in co-ordination. But a more sober and somewhat disturbing view of the process stems from the implication of rising complexity within the unit of division of labour. A growing number of interrelated traits brings about the danger of sliding down into mediocrity and easy lock-in into suboptimal peaks: an event which has been dubbed by some authors a complexity catastrophe. This is far from being an unlikely occurrence in an economic environment. It is, in fact sufficient to observe that development fosters lengthening of production processes through more roundabout, mechanised technologies. Thus, as phases or firms get trapped into sub-optimality, the need to decompose, to spin-off parts to ease the burden of problem solving becomes greater: in this sense the complexity stalemate is a triggering device to further division of labour.

The framework provided by Landscape theory and the NKC model gives us some new and partially unexplored perspectives for future research in understanding the impact of distributed innovative activities into the division of labour and co-ordination. The model, as well as an increasing experimental evidence induce to believe that localised learning which takes place within any organisation, has endogenous limits : this provides a cognitive explanation of the fact that to co-ordinate the activities of large groups "formal" institutions arise within organisations. 


\section{References}

Allais M. (1953). Le comportement de l' homme rationel devant le risque: Critique des postulats et axiomes de l'Ecole Américaine, Econometrica, 21, 503-46.

Arrow K. J. (1974). The Limits of Organisations. New York, Norton.

Axelrod R. (1984). The Evolution of Cooperation, New York, Basic Books.

Cohen M. D. (1991). Individual learning and organizational routine: Emerging connections. Organization Science, 2 (1), 135-139.

Cohen, M. D. and Bacdayan P. (1995). Organizational Routines are Stored as Procedural Memory: Evidence from a Laboratory Study. Organization Science, $5,554-568$.

Cyert, R. M. and March J.G.(1992). A Behavioral Theory of the Firm, 2nd edition. Cambridge, MA. Blackwell Publishers.

Egidi M. (1992). Organizational Learning and the Division of Labour, in Egidi M, Marris R. (eds) Economics, Bounded Rationality and the Cognitive Revolution, Aldershot, Edward Elgar.

Egidi M. (1996) Routines, Hierarchies of Problems , Procedural Behaviour: Some Evidence from Experiments in Arrow K. J., Colombatto E., Perlman M. and Schmidt C. (eds.) The Rational Foundations of Economic Behaviour, MacMillan, London and St. Martin's Press, New York, pp. 303-333

Egidi M., Narduzzo A. (1996). The Emergence of Path dependent behaviors in cooperative context CEEL Working Papers, forthcoming in International Journal of Industrial Organization,. North Holland.

von Hayek F. A. (1980). Economics and Knowledge, in Individualism and Economic Order (Reprint of 1948 first printing) Chicago, The University of Chicago Press

Henderson, R. M. (1992), Technological Change and the Management of Architectural Knowledge. In Kochan, T. A. and Useem M. (eds),Transforming Organizations,. Oxford University Press..

Holland J. H. (1975). Adaptation in Natural and Artificial Systems. Ann Arbor, University of Michigan Press.

Holland J. H., Holyoak K, J., Nisbett R.E., Thagard P.R. (1988). Induction, Processes of Inference, Learning, and Discovery, Cambridge (Mass) MIT Press 
Kahneman D., Tversky A. (1986). Rational choice and the Framing of Decisions in Hogart R. M., Reder M. W.(eds.). Rational choice - The Contrast between Economics and Psychology, Chicago, The University of Chicago Press.

Kauffman S. A. (1989)., Adaptation on Rugged Fitness Landscapes, in Stein D(ed.). Lectures on the Science of Complexity. Reading, Addison Wesley Longman

Kauffman S.A., Johnsen S. (1992), Coevolution to the Edge of Chaos. in Langton et alii (eds.) Artificial Life II. Reading. Addison Wesley Longman

Luchins A.S. (1942). Mechanization in Problem-Solving, Psychological Monograph 54, $1-95$.

March J. G. (1988), Decisions and Organisations, Oxford, Basil Blackwell.

Nelson R. R. and Winter S. (1982), An Evolutionary Theory of Economic Change, Cambridge (Mass), The Belknap Press.

Ricottilli M. (1994), Technical Progress, Innovative Activity, and Development Policies, International. Journal Of Technology Management, 9, 297-313

Simon H. A. (1957), Models of Man,New York, Wiley

Simon H. A. (1963), Problem Solving Machines, International Science and Technology, 3,48-62.

Simon H.A. and Newell A. (1972), Human Problem Solving, Englewood Cliffs, Prentice-Hall

Simon H. A. (1979), Rational Decision Making in Business Organization, American Economic Review, 69, 493-513

Weisberg R. (1980) Memory: Thought and Behaviour, New York: Oxford University Press

Winter S. G. (1982), An Essay on the Theory of Production, S. H. Hymans (ed.), Economics and the World Around It, Ann Arbor, Michigan University Press 